\title{
Verbesserung der Lebensqualität von Patienten mit Psoriasis unter Therapie mit Adalimumab
}

\author{
Improvement of Quality of Life in Patients with Psoriasis Being Treated \\ with Adalimumab
}

Autoren

Institut
F. Dörfler, A.Schöberl, U. Amon

PsoriSol Hautklinik GmbH
Bibliografie

Dol http://dx.doi.org/ 10.1055/s-0029-1244166

Online-Publikation: 26. 5. 2010

Akt Dermatol 2010; 36:

305-308 ๑ Georg Thieme

Verlag KG Stuttgart · New York ISSN 0340-2541

\section{Korrespondenzadresse}

Prof. Dr. med. habil.

\section{Ulrich Amon}

PsoriSol Hautklinik GmbH Fachklinik für Dermatologie und Allergologie

Mühlstraße 31

91217 Hersbruck

profamonklinik@aol.com

\section{Zusammenfassung \\ $\nabla$}

Die TNF- $\alpha$-Antagonisten, wie der monoklonale Antikörper Adalimumab, sind in der Psoriasistherapie inzwischen eine effektive Erweiterung der bisherigen Therapieschemata, insbesondere für Patienten, die auf die klassischen Behandlungsstrategien nicht ausreichend ansprechen bzw. die Kontraindikationen hierfür aufweisen. In unserer Studie wurde die Veränderung der Lebensqualität von 33 Patienten mit mittelschwerer bis schwerer Psoriasis vulgaris, z.T. mit Gelenkbeteiligung, vor sowie zum Zeitpunkt der letzten Injek-

\section{Hintergrund}

Die Lebensqualität von Patienten mit Psoriasis vulgaris ist nach aktueller Studienlage im Vergleich zur Normalbevölkerung zum Teil massiv eingeschränkt mit erhöhten Prävalenzraten für Depression, einhergehend mit Suizidgedanken, Ängsten, Wut und sozialem Rückzug [1,2]. Angesichts dieser Tatsache erscheint es umso dringlicher, den Patienten durch geeignete therapeutische Maßnahmen zu einer verbesserten Lebensqualität zu verhelfen. Die Verordnung von Biologika stellt als Second-line-Therapie mittlerweile eine fest etablierte, leitlinienorientierte Behandlung für mittelschwere bis schwere Formen der Plaque-Psoriasis dar [3-6]. In der folgenden Studie soll die Frage beantwortet werden, welchen Einfluss die stationär eingeleitete Verordnung eines TNF- $\alpha$-Inhibitors, im vorliegenden Fall Adalimumab, auf die Lebensqualität von Patienten mit Psoriasis vulgaris hat und wie sich unter den stark reglementierten Bedingungen des GKV-Bereichs eine poststationäre Empfehlung zur Weiterbehandlung auswirkt. tion mit Adalimumab mittels DLQI-Fragebogen retrospektiv erhoben. Es zeigte sich eine deutliche Reduktion des DLQI von initial durchschnittlich 20,5 auf 6,4 und somit eine bemerkenswerte $\mathrm{Zu}$ nahme der Lebensqualität der Patienten, wobei insgesamt 24 (72,7\%) der eingeschlossenen Patienten die Behandlung als „sehr erfolgreich“ einstuften und 20 (62,5\%) Patienten keinerlei Nebenwirkungen bemerkten. Bei neun (27,3\%) Patienten bestanden Probleme bei der ambulanten Weiterverordnung des Medikaments. Adalimumab führt zur deutlichen Verbesserung der Lebensqualität bei Patienten mit Psoriasis.

\section{Patienten und Methoden}

Der Dermatology Life Quality Index (DLQI) gilt als sehr gut validiertes Instrument zur Evaluierung der Lebensqualität bei verschiedenen Hauterkrankungen [7,8].

In unserer Studie wurden bei 33 Patienten ( $\bullet$ Abb. 1) mit mittelschwerer bis schwerer Plaque-Psoriasis, bei denen stationär die Einleitung einer Therapie mit Adalimumab (Humira ${ }^{\circledR}$ ) erfolgte, retrospektiv der DLQI erhoben.

Es erfolgte ein Fragebogenmailing, bei dem die Patienten gebeten wurden, die Lebensqualität sowohl vor Therapiebeginn retrospektiv als auch zum Zeitpunkt der Befragung aktuell differenziert zu bewerten. Bei 23 (69,7\%) Patienten bestand zusätzlich eine Osteoarthropathia psoriatica.

Dosierung und Behandlungsfrequenz der subkutanen Injektionen von Adalimumab erfolgten gemäß den Empfehlungen des Herstellers.

Neun, d. h. 27,3\% der Patienten, erhielten zusätzliche systemische Therapien, acht (24,3\%) Patienten Methotrexat, ein (3\%) Patient Ciclosporin A. Für $43 \%$ (13 Patienten) betrug die Therapiedauer $>12$ Monate, für $10 \%$ (3 Patienten) 6 bis 12 Monaten, für 43\% (13 Patienten) $3-<6$ Monate und für 


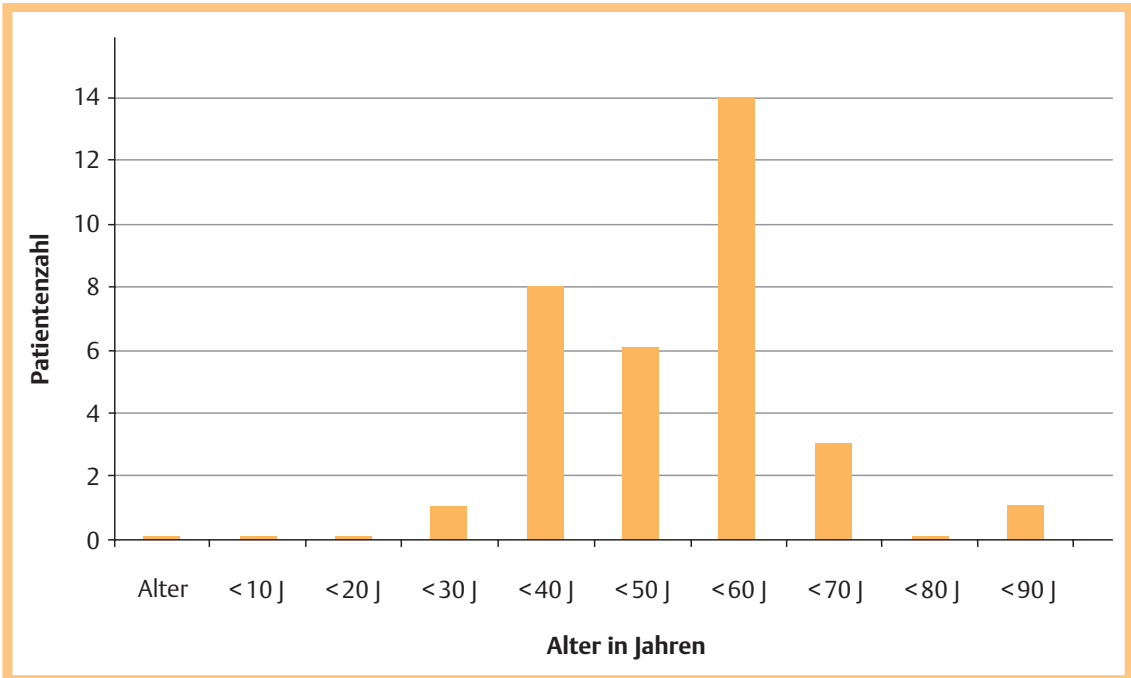

Abb. 1 Altersverteilung.

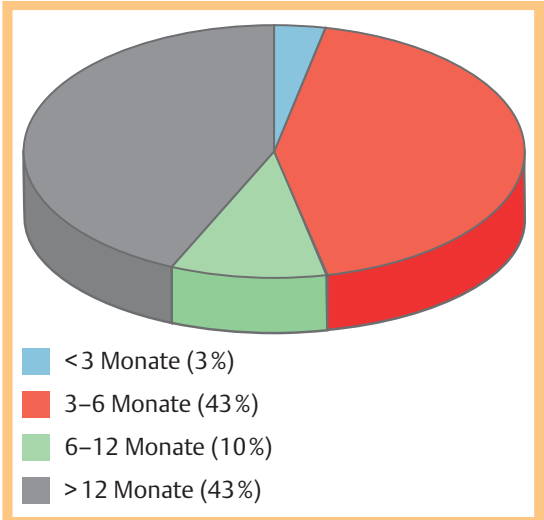

Abb. 2 Dauer der Adalimumab-Behandlung zum Zeitpunkt der Erhebung. Notabene: Drei Patienten mit unbekannter Behandlungsdauer ausgeschlossen.

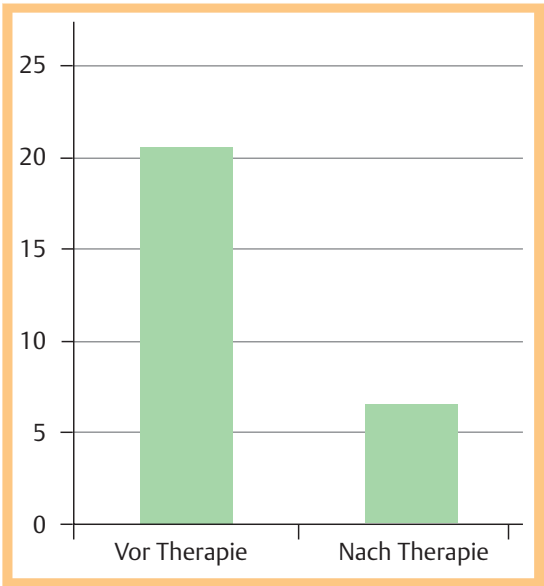

Abb. 3 Gesamt-DLQI vor und nach Therapie.

3\% (1 Patient) weniger als 3 Monate ( Abb. 2). Bei 3 Patienten war die Therapiedauer nicht zu eruieren.

\section{Ergebnisse}

$\nabla$

Der durchschnittliche DLQI vor Einleitung der Behandlung mit Adalimumab betrug 20,5, zum Zeitpunkt der letzten Injektion hingegen 6,4, sodass eine deutliche Abnahme des Scores und somit eine erhebliche Verbesserung der Lebensqualität insgesamt zu verzeichnen war ( $\mathbf{A b b} .3$ und $\mathbf{4})$.
24 Patienten (72,7\%) beurteilten selbst den Therapieerfolg als „sehr gut“, 5 Patienten, d.h. 15,2\%, sahen einen „mäßigen Erfolg“ und 3 Patienten, entsprechend 9\%, „keine Verbesserung“. Ein Patient ( $3 \%$ ) gab an, unter der Therapie eine Verschlechterung erfahren zu haben ( $\bullet$ Abb. 5).

Bei 20 (62,5\%) Patienten traten keinerlei Nebenwirkungen auf (๑ Abb. 6).

Ansonsten wurden als Nebenwirkung bei fünf $(15,6 \%)$ Patienten Laborveränderungen, bei drei $(9,4 \%)$ Patienten gastrointestinale Beschwerden, bei einem (3\%) Patient Infektionen und bei fünf (15,6\%) Patienten andere diskrete Nebenwirkungen, wie Kopfschmerzen, Müdigkeit am Tag der Spritze, Haarausfall und Gewichtszunahme, angegeben.

Bei 24 Patienten (72,7\%) ergaben sich keinerlei Probleme bei der ambulanten Weiterverordnung $(\bullet$ Abb. 7).

Bei den restlichen neun Patienten $(27,3 \%)$ waren Verordnungsprobleme einerseits aus „Budget-Gründen“ des niedergelassenen Arztes (66,6\%) und andererseits aufgrund unzureichender Erfahrungen mit dem Medikament durch den niedergelassenen Arzt $(33,3 \%)$ zu verzeichnen.

\section{Diskussion}

$\nabla$

Adalimumab ist ein Vertreter der Gruppe der „Biologika“. Es handelt sich um einen rekombinanten, humanen, monoklonalen Antikörper gegen das Schlüsselmolekül TNF- $\alpha$, der unter anderem für die Second-line-Therapie bei Erwachsenen zur Behandlung der mittelschweren bis schweren Plaque-Psoriasis und der aktiven und fortschreitenden Psoriasis-Arthritis zugelassen ist. Als Nebenwirkung können eine erhöhte Infektanfälligkeit, Veränderungen beim Blutbild, Leberwerten etc., Kopfschmerzen, Hautausschlag und gastrointestinale Beschwerden auftreten. Kontraindikationen sind eine aktive Tuberkulose, schwere und opportunistische Infektionen, Schwangerschaft und Stillzeit, eine mäßigbis schwergradige Herzinsuffizienz sowie der Einsatz bei Kindern und Jugendlichen unter 18 Jahren. Die Therapiekosten belaufen sich normalerweise auf etwa $870 €$ pro Spritze.

Die Wirksamkeit von Adalimumab konnte in einer randomisierten, doppelblinden Phase-III-Studie mit 1212 Patienten über eine Studiendauer von 52 Wochen nachgewiesen werden [9]: Eine 75\%-PASI-Reduktion in $71 \%$ der Patienten nach Woche 16 wurde hierbei beschrieben. 


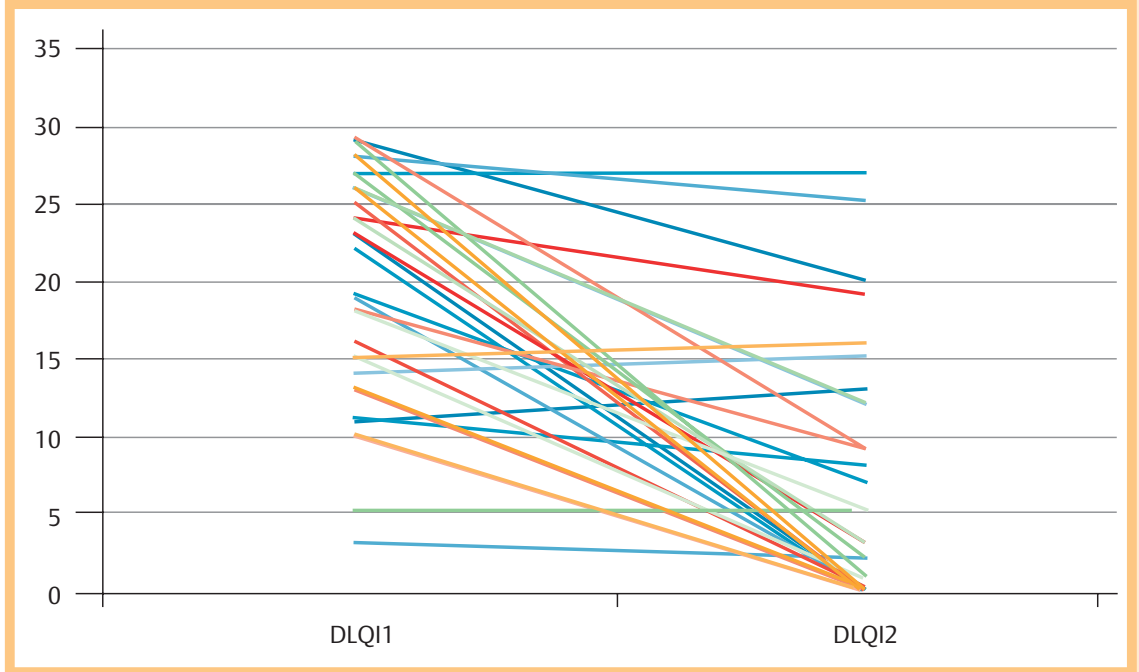

Abb.4 Individuelle DLQI-Verläufe vor und nach Therapie.

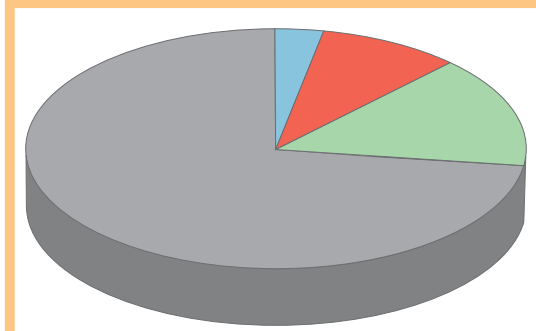

Abb. 5 Therapieerfolg aus Patientensicht.

verschlechtert $(3,03 \%)$

mäßig (15,15\%)

kein $(9,09 \%)$

sehr gut $(72,73 \%)$

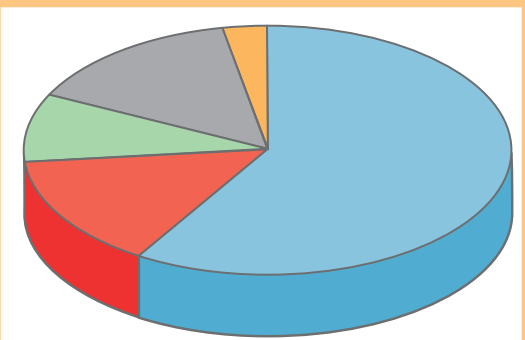

Abb. 6 Nebenwirkungen aus Patientensicht. Notabene: Ein Patient wegen „unbekannt“ ausgeschlossen. Zwei Patienten mit mehreren UAW.

Magen-Darm Beschwerden (3)

Laborwertänderungen (5)

Infektionen (1)

Sonstige (5)

Keine (20)
Die Patienten unserer Retrospektivanalyse sind mit dieser Behandlungsform insgesamt sehr zufrieden. Die Lebensqualität hat sich hochsignifikant verbessert.

Saurat et al. beschreiben außerdem eine signifikante Verbesserung der Lebensqualität unter Adalimumab im Vergleich zu Methotrexat $15 \mathrm{mg}$ p. o. und im Vergleich zu Placebo in einer doppelblinden, randomisierten Phase-III-Studie mit 271 Patienten über eine Studiendauer von 16 Wochen [10]. Auch Revicki u. Mitarb. zeigten signifikante Verbesserungen des DLQI in einer Multizenter-Phase-III-Studie unter der Therapie mit Adalimumab über 16 Wochen im Vergleich mit Placebo [11].
Die vorliegende Retrospektivanalyse an eigenen Patienten zeigt eine hohe Akzeptanz und Effektivität dieser Behandlungsform. Insbesondere die Bewertung des Therapieerfolgs aus Patientensicht fällt sehr positiv aus. Die deutlichen Alltagseinschränkungen der Patienten mit mittelschwerer bis schwerer Psoriasis [8] bessern sich signifikant unter der Biologic-Therapie und verhelfen den Patienten zu einer gesteigerten Lebensqualität. Die vorliegenden Daten bestätigen insofern die Daten von Reivicki u. Mitarb. [11].

Eine Schwäche unserer Studie ist sicherlich die fehlende Vergleichsgruppe. Daher kann hier nicht mit letzter Sicherheit entschieden werden, ob die Verbesserung der Lebensqualität in direktem Zusammenhang mit der Adalimumab-Therapie steht oder auch durch die (teilweise systemische) Begleitmedikation bedingt sein kann. Allerdings ist anzumerken, dass eine Adalimumab-Therapie nur bei Patienten eingeleitet wurde, die unter klassischer topischer und/oder systemischer Therapie nur unzureichende Symptomkontrolle zeigten. Es kann also durchaus von einem Effekt der TNF-alpha-Antikörper ausgegangen werden. Ein weiteres Problem ist die retrospektive Beurteilung der Lebensqualität. Eine exakte Beurteilung der Lebensqualität in der Vergangenheit fällt eventuell einigen Patienten schwer. Durch die einzelnen, sehr spezifischen Items des DLQI lassen sich „gefühlte“ Unterschiede in der Lebensqualität dennoch objektivieren. Eine prospektive Analyse wäre in künftigen Untersuchungen jedoch wünschenswert.

Nach den derzeit vorliegenden Daten der Behandlung verschiedener immunologischer Erkrankungen mit Adalimumab kann die Behandlung als insgesamt sehr gut verträglich eingestuft werden [12]. Auch in unserer Untersuchung hat die große Mehrheit der Patienten (20 Patienten [62,5\%]) die Injektionstherapie gut vertragen.

Immerhin über ein Viertel der befragten Patienten (neun Patienten $(27,3 \%)$ berichtete über Probleme der poststationären Verordnung von Adalimumab. Die Verordnungen fanden ausschließlich bei niedergelassenen Dermatologen statt. Die hohe Zahl überrascht insofern etwas, als die stationäre Verordnung im Sinne eines standardisierten Verfahrens stets mit den einweisenden Hautärzten abgestimmt wird. Eine nach den vorliegenden Daten durchgeführte aktuelle Umfrage unter 122 bayerischen Dermatologen Ende 2009 zeigte bei einer Rücklaufquote von 42\%, dass annähernd 90\% der Kollegen einen Regress bei der „Verordnung teurer Medikamente“ sehr befürchten oder befürchten. Im direk- 


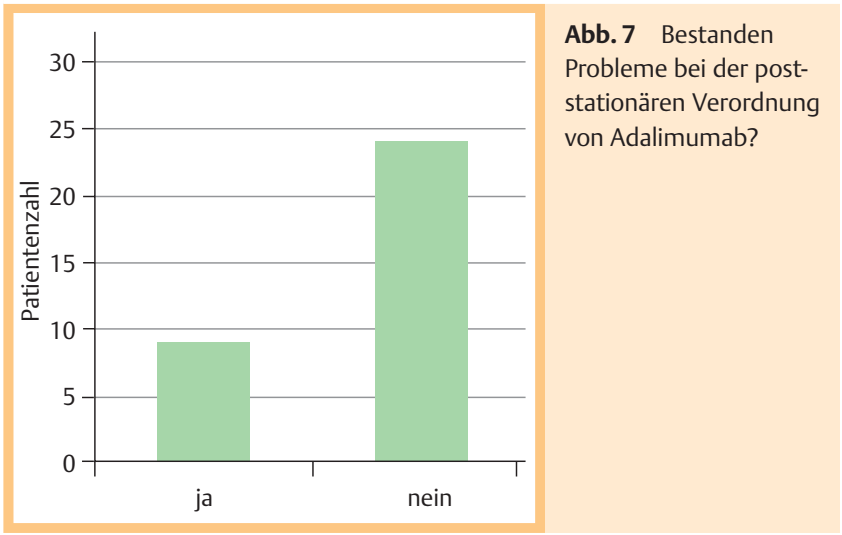

ten Vergleich zu anderen Systemtherapeutika waren Biologika dann in der abgefragten Verordnungshäufigkeit deutlich unterrepräsentiert (eigene nicht publizierte Ergebnisse). Sollte sich die ambulante Versorgungssituation mittelfristig nicht insofern ändern, dass Second-line-Therapeutika bei leitliniengerechter Verordnung und lückenloser Dokumentation automatisch einem Regressrisiko entzogen werden, steht zu befürchten, dass beispielsweise Biologika bei Psoriasis trotz der nachgewiesenen Effektivität nicht den hohen Marktdurchdringungsgrad erhalten werden, der aufgrund der medizinischen Notwendigkeit eigentlich zu erwarten wäre.

\section{Fazit}

Der TNF- $\alpha$-Antagonist Adalimumab stellt eine wirkungsvolle Erweiterung der bisherigen Behandlungsstrategien in der Psoriasistherapie dar und ist zugelassen zur Behandlung der mittelschweren bis schweren Plaque-Psoriasis und der aktiven und fortschreitenden Psoriasis-Arthritis. Adalimumab ist somit insbesondere eine hilfreiche Alternative für Patienten, die auf die klassischen Therapieschemata wie Lichttherapie, Methotrexat oder Ciclosporin A nicht ausreichend ansprechen. Bei leitliniengerechter Anwendung und unter Berücksichtigung des individuellen Risikoprofils ist eine signifikante Verbesserung der krankheitsspezifischen Lebensqualität bei überschaubarem Nebenwirkungsprofil zu erwarten. Langzeitstudien bleiben jedoch abzuwarten. Das Verordnungsverhalten ist derzeit abhängig von gesundheitspolitischen Rahmenbedingungen.

\section{Abstract}

\section{Improvement of Quality of Life in Patients with Psoriasis Being Treated with Adalimumab}

$\nabla$

Monoclonal antibodies against TNF- $\alpha$, such as adalimumab, have been successfully implemented with treatment strategies for patients suffering from moderate to severe psoriasis who do not respond to or demonstrate contraindications for conventional treatment. We performed a retrospective analysis in 33 patients having received adalimumab for severe plaque-type psoriasis with or without arthritis focusing on quality of life using the DLQI questionnaire. During treatment with adalimumab the mean DLQI value significantly dropped from 20.5 to 6.4 , which means an excellent increase in quality of life. 24 (72.7\%) patients described the treatment to be „very successful“ and 20 (62.5\%) patients did not report any side effects. However, due to different reasons nine $(27.3 \%)$ patients got problems with continuous prescription of the drug. Adalimumab has a strong positive impact on improvement of quality of life in patients with severe psoriasis.

\section{Literatur}

1 Gupta MA, Gupta AK. Depression and suicidal ideation in dermatology patients with acen, alopecia areata, atopic dermatitis and psoriasis. Br J Dermatol 1998; 139: 846-850

2 Gottlieb AB, Chao C, Dann F. Psoriasis comorbidities. J Dermatolog Treat 2008; 19: 5-21

3 Mease PJ, Ory P, Sharp JT, Ritchlin CT et al. Adalimumab for long-term treatment of psoriatic arthritis: 2-year data from the Adalimumab Effectiveness in Psoriatic Arthritis Trial (ADEPT). Ann Rheum Dis 2009; 68: $702-709$

4 Papoutsaki M, Costanzo A, Chimenti MS, Chimenti S. Adalimumab for the treatment of severe psoriasis and psoriatic arthritis. Expert Opin Biol Ther 2008; Mar : $363-370$

5 Griffiths CE, Iaccarino L, Naldi $L$ et al. Psoriasis and psoriatic arthritis: immunological aspects and therapeutic guidelines. Clin Exp Rheumatol 2006; 24 (1 Suppl 40): $72-78$

6 Pathirana D, Ormerod AD, Saiag P et al. European S3-guidelines on the systemic treatment of psoriasis vulgaris. J Eur Acad Dermatol Venereol 2009; 23 (Suppl 2): $1-70$

7 Finlay AY, Khan GK. Dermatology Life Quality Index (DLQI) - a simple practical measure for routine clinical use. Clin Exp Dermatol 1994; 19: $210-216$

8 Katugampola RP, Lewis VJ, Finlay AY. The Dermatology Life Quality Index: assessing the efficacy of biological therapies for psoriasis. $\mathrm{Br} \mathrm{J}$ Dermatol 2007; 156: 945-950

9 Menter A, Tyring SK, Gordon K et al. Adalimumab therapy for moderate to severe psoriasis: A randomized, controlled phase III trial. J Am Acad Dermatol 2008; 58: 106 - 115

10 Saurat JH, Stingl G, Dubertret $L$ et al. Efficacy and safety results from the randomized controlled comparative study of adalimumab vs. methotrexate vs. placebo in patients with psoriasis (CHAMPION). Br J Dermatol 2008; 158: $558-566$

11 Revicki DA, Willian MK, Menter A et al. Impact of adalimumab treatment on health-related quality of life and other patient-reported outcomes: results from a 16-week randomized controlled trial in patients with moderate to severe plaque psoriasis. J Dermatolog Treat 2007; 18: $341-350$

12 Burmester GR, Mease P, Dijkmans BAC et al. Adalimumab safety and mortality rates from global clinical trials of six immune-mediated inflammatory diseases. Ann Rheum Dis 2009; 68: 1863-1869 